\title{
Solid-Fuel Regression Rate for Standard-Flow Hybrid Rocket Motors*
}

\author{
Takakazu MORITA**, Saburo YUASA***, Shigeru YAMAGUCHI**** \\ and Toru SHIMADA $* * * * *$ \\ **Department of Aeronautics and Astronautics, Tokai University \\ 4-1-1 Kitakaname, Hiratsuka, Kanagawa 259-1292, Japan \\ E-mail: morita@tokai-u.jp \\ ***Department of Aerospace Engineering, Tokyo Metropolitan University \\ 6-6 Asahigaoka, Hino, Tokyo 191-0065, Japan \\ ****Department of Physics, Tokai University \\ *****Institute of Space and Astronautical Science, JAXA
}

3-1-1 Yoshinodai, Chuo, Sagamihara, Kanagawa 252-5210, Japan

\begin{abstract}
Marxman's diffusion-limited analysis of hybrid rocket combustion has been often used to investigate various combustion problems in hybrid rocket motors. This analysis was developed on the basis of the Reynolds analogy in turbulent boundary layers. This analogy assumes that both molecular and turbulent Prandtl numbers are equal to one. In the present study, a semi-empirical correlation between the Stanton number and the skin-friction coefficient in a turbulent boundary layer was obtained. This is applicable to hybrid rocket combustion, and also includes the effects of the Prandtl numbers variation. Using this correlation, a fuel regression rate equation for standard-flow hybrid rocket motors was obtained, and its characteristics were examined. In addition, the calculated regression rate characteristics were compared with the experimental data from the laboratory-scale hybrid rocket motors that used gaseous oxygen (GOX) as oxidizer and polymethylmethacrylate (PMMA) as fuel.
\end{abstract}

Key words: Hybrid Rocket, Fuel Regression Rate, Turbulent Boundary Layer, Diffusion-Limited Analysis, Polymethylmethacrylate (PMMA), Prandtl Number

\section{Introduction}

Marxman et al. proposed a regression rate expression for diffusion-limited combustion in the turbulent boundary layer of hybrid rocket motors ${ }^{(1-3)}$. This theory is well-known to anyone working in or studying hybrid rocket propulsion, and it has been used frequently in order to investigate various problems related to combustion in hybrid rockets. For example, Karabeyoglu et al. investigated the oscillatory combustion in hybrid rockets using this theory ${ }^{(4)}$. According to Marxman's diffusion-limited analysis, the mass flux exponent of hybrid rocket propellants is $0.8^{(1)}$. However, based on many experiments, the mass flux exponents are not equal to 0.8 . These differences are caused by the following factors: swirling flow, flow impingement, radiation, slow chemical reactions in the gas phase, etc. These factors were not considered in this study. We treated only diffusion-limited boundary layer combustion under standard-flow conditions. In the analysis proposed by Marxman et al., the molecular and turbulent Prandtl numbers are both assumed to be one, respectively. On the other hand, some researchers proposed theoretical regression rate expressions that allow for the Prandtl numbers to vary. For example, Boardman derived a theoretical

Received 6 Feb., 2012 (No. 12-0096)

DOI: 10.1299/jtst.7.387]

Copyright $(C) 2012$ by JSME 
regression rate using the Chilton-Colburn analogy ${ }^{(5)}$. However, the analogy neglects the effects of the temperature deviation caused by the flame in a turbulent boundary layer. Here we have treated precisely those effects that were neglected in the previous studies. From many experimental results, Yuasa et al. suggested that diffusion-limited combustion is dominant in the gas-phase reactions of polymethylmethacrylate (PMMA) with gaseous oxygen $(\mathrm{GOX})$ in laboratory-scale hybrid rocket engines ${ }^{(6)}$. In this case, the mass flux exponent is slightly less than $0.8^{(7-9)}$. Although various factors are considered as the cause, we investigated effects on the Prandtl numbers in particular ${ }^{(10-11)}$.

\section{Nomenclature}

$a:$ regression rate coefficient

$B$ : blowing parameter

$B_{a}, B_{t}$ : aerodynamic and thermal blowing parameters

$C_{1}, C_{2}, C_{3}$ : defined by Eq. (17), Eq. (18), and Eq. (19), respectively

$c_{c}:$ specific heat of condensed phase

$c_{f}:$ skin-friction coefficient

$c_{f, 0}:$ skin-friction coefficient with no blowing

$c_{H}: \quad c_{H}=\left(\operatorname{Pr} / \operatorname{Pr}_{t}\right) \operatorname{Re}_{x}(\delta / x) \kappa^{2} \ell$

$c_{p}:$ constant-pressure specific heat

$G$ : mass flux

$G^{*}: \quad$ nondimensional mass flux; $G^{*}=G / G_{r e f}$

$h_{v}$ : total heat for pyrolyzing solid fuel from its initial temperature

$I_{H}:$ defined by Eq. (6)

$k$ : defined by Eq. (33)

$\ell$ : defined by Eq. (13)

$\ell^{\prime}: \quad \ell^{\prime}=\ell /(1+\ell)$

$\dot{m}^{*}:$ nondimensional regression rate; $\dot{m}^{*}=\rho_{c} \dot{r} / G_{r e f}$

$n:$ mass flux exponent

$O / F$ : oxidizer-to-fuel mass ratio

$P r, P r_{t}: \quad$ molecular and turbulent Prandtl numbers

$Q_{s}:$ surface heat release ( $Q_{s}<0$ :endothermic, $Q_{s}>0$ :exothermic)

$q: \quad$ defined by Eq. (33)

$\dot{q}_{s+}$ : wall heat flux from the flame to the fuel surface

$\dot{q}_{s^{-}}$: wall heat flux from the fuel surface to the solid phase

$R e_{x}$ : local Reynolds number; $R e_{x}=u_{e} x / \nu=G x / \mu$

$R e_{x, r e f}: \quad R e_{x, r e f}=G_{r e f} x / \mu$

$\dot{r}: \quad$ fuel regression rate

$S t:$ Stanton number

$T:$ temperature

$u$ : velocity component in $\mathrm{x}$-direction

$x$ : rectangular coordinate; distance parallel to surface

$Y_{o x}$ : mass fraction of oxidizer

$y$ : rectangular coordinate; distance perpendicular to surface

$\alpha: \quad$ thermal diffusivity

$\beta: \quad$ defined by Eq. (A2)

$\beta_{0}:$ constant of integration

$\Delta h:$ enthalpy difference between the flame and the surface

$\delta: \quad$ velocity boundary-layer thickness 


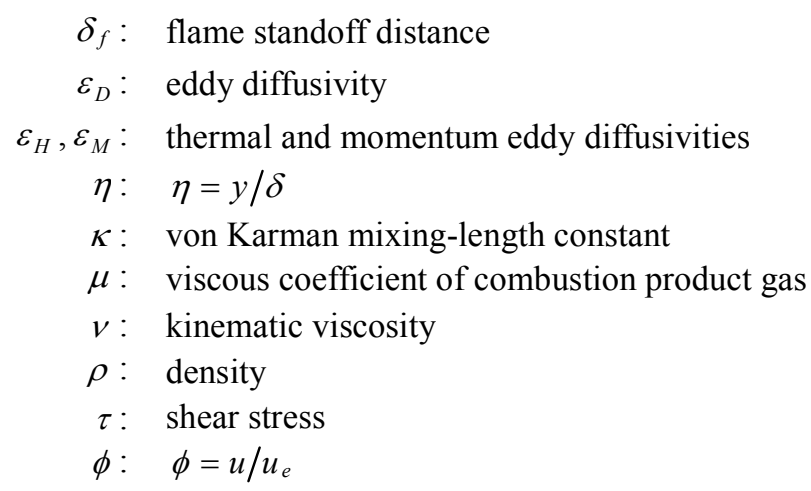

\section{Subscripts}

c: condensed phase

e: boundary-layer edge

f: flame

ox: oxidizer

ref: reference quantities

s: fuel surface

\section{Analogy Between Heat and Mass Transfer}

Derivation of a new correlation between the Stanton number and the skin friction coefficient is presented in this section. In a turbulent boundary layer, the total shear stress and the total heat flux are represented by the following equations:

$$
\begin{aligned}
& \tau=\rho\left(v+\varepsilon_{M}\right) \frac{\partial u}{\partial y}, \\
& \dot{q}=\rho c_{p}\left(\alpha+\varepsilon_{H}\right) \frac{\partial T}{\partial y} .
\end{aligned}
$$

The following assumption is usually used for analogies between heat and momentum transfer in a turbulent boundary layer:

$$
\frac{\dot{q}}{\dot{q}_{s+}}=\frac{\tau}{\tau_{s}} .
$$

Substituting Eqs. (1) and (2) into Eq. (3), we can obtain the following equation:

$$
\frac{\partial T}{\partial y}=\frac{\dot{q}_{s+}}{\tau_{s} c_{p}} \frac{v+\varepsilon_{M}}{v / P r+\varepsilon_{M} / P r_{t}} \frac{\partial u}{\partial y} .
$$

Integrating the above equation from the fuel surface to the flame edge, the result shows:

$$
T_{f}-T_{s}=\frac{\dot{q}_{s+}}{\tau_{s} c_{p}} u_{e} I_{H},
$$

where $I_{H}=\int_{0}^{\delta_{f} / \delta} \frac{v+\varepsilon_{M}}{v / P r+\varepsilon_{M} / P r_{t}} \frac{d}{d \eta}\left(\frac{u}{u_{e}}\right) d \eta$.

The local Stanton number can be written in terms of the heat flux to the fuel surface as follows:

$$
S t=\frac{\dot{q}_{s+} /\left(T_{f}-T_{s}\right)}{\rho_{f} u_{f} c_{p}} .
$$

Using the local skin friction coefficient, the shear stress at the fuel surface can be expressed as in Eq. (8): 


$$
\tau_{s}=\frac{1}{2} c_{f} \rho_{e} u_{e}^{2}
$$

Substituting Eqs. (7) and (8) into Eq. (5), we can obtain a new correlation between the local Stanton number and the local skin friction coefficient as follows:

$$
S t=\frac{c_{f}}{2} \frac{\rho_{e} u_{e}^{2}}{\rho_{f} u_{f}^{2}} \frac{1}{I_{H}} .
$$

In particular, if both the molecular and the turbulent Prandtl numbers are equal to one, $I_{H}$ becomes $u_{f} / u_{e}$. In this case, the above equation can be simplified, as shown below:

$$
S t=\frac{c_{f}}{2} \frac{\rho_{e} u_{e}^{2}}{\rho_{f} u_{f}^{2}} .
$$

This correlation is the same as the result obtained by Marxman et al.

\section{Solid-Fuel Regression Rate}

Lengelle proposed the following equation as the velocity distribution with blowing in turbulent boundary layers ${ }^{(12)}$ :

$$
\phi=\frac{u}{u_{e}}=\eta^{\ell}
$$

where $\eta=y / \delta$,

$$
\begin{aligned}
& \ell=\ell_{0} \frac{1}{B_{a}}\left(1+B_{a}\right) \ln \left(1+B_{a}\right), \\
& \ell_{0}=\frac{1}{7} .
\end{aligned}
$$

In Eqs. (11)-(14), $B_{a}$ is an aerodynamic blowing parameter, and $u_{e}$ is the gas velocity in the main stream, respectively. Karabeyoglu found that the calculation accuracy of the velocity distribution obtained by Marxman et al. becomes worse with comparison to Eq. (11) as the blowing from the fuel surface becomes stronger ${ }^{(13)}$. We therefore used this velocity distribution in order to obtain a theoretical regression rate for hybrid rocket combustion. Equation (6) can be integrated approximately using Eq. (11). Consequently, we can obtain the solution, as shown below. Detailed calculation to deduce the solution is represented in the appendix.

$$
I_{H}=\int_{0}^{\delta_{f} / \delta} \frac{v+\varepsilon_{M}}{v / P r+\varepsilon_{M} / \operatorname{Pr}_{t}} \frac{d}{d \eta}\left(\frac{u}{u_{e}}\right) d \eta=\phi_{f} F_{R},
$$

where $F_{R}=P r_{t}+\left(P r-P r_{t}\right)\left\{\frac{1}{C_{1}}+\frac{C_{2}}{\left(1-\ell^{\prime}\right) c_{H} \ell^{\prime} \phi_{f}}\right\}$,

$$
C_{1}=1+c_{H} \phi_{f}^{1 / \ell^{\prime}},
$$

$$
C_{2}=-\frac{1}{C_{1}^{1-\ell^{\prime}}}+\left(\frac{\beta_{0}}{1+\beta_{0}}\right)^{1-\ell^{\prime}}+\frac{1-\ell^{\prime}}{1+\ell^{\prime}} \frac{C_{3}}{1+\beta_{0}},
$$

$$
C_{3}=\left\{\beta_{0}\left(1+\beta_{0}\right)\right\}^{-\ell^{\prime}} \text {, }
$$

$$
\phi_{f}=\frac{u_{f}}{u_{e}},
$$

$$
\ell^{\prime}=\frac{\ell}{1+\ell},
$$




$$
c_{H}=\left(P r / \operatorname{Pr}_{t}\right) \operatorname{Re}_{x}(\delta / x) \kappa^{2} \ell
$$

$\delta / x$ was calculated using the result obtained by Marxman et al ${ }^{(2)}$. In the steady-state, the energy balance equation at the fuel surface is expressed by the following equation:

$$
\dot{q}_{s-}=\rho_{c} \dot{r} Q_{s}+\dot{q}_{s+} .
$$

From Eqs. (7) and (8), we can obtain the wall heat flux from the flame to the fuel surface.

$$
\dot{q}_{s+}=G \frac{c_{f}}{2} \frac{\Delta h}{I_{H}}
$$

The heat conduction equation in the solid phase is expressed as:

$$
\dot{r} \frac{d T}{d x}-\alpha_{c} \frac{d^{2} T}{d x^{2}}=0 .
$$

Solving the above differential equation and then deducing the wall heat flux using the solution, the result shows:

$$
\dot{q}_{s-}=\rho_{c} \dot{r}\left\{c_{c}\left(T_{s}-T_{0}\right)\right\} \text {. }
$$

Substituting Eqs. (24) and (26) into Eq. (23),

$$
B_{a}=\frac{\Delta h}{c_{c}\left(T_{s}-T_{0}\right)+Q_{s}} \frac{1}{I_{F}}=\frac{u_{e}}{u_{f}} \frac{\Delta h}{h_{v}} \frac{1}{F_{R}},
$$

where $h_{v}=c_{c}\left(T_{s}-T_{0}\right)+Q_{s}$

Aerodynamic blowing parameter, $B_{a}$, is defined as:

$$
B_{a} \equiv \frac{\rho_{c} \dot{r}}{G\left(c_{f} / 2\right)} .
$$

In this study, the definition of $B_{t}$ must be modified, because the present model allows for variation of the Prandtl numbers. If $B_{t}$ is defined as the following equation, $B_{t}$ is equal to $B_{a}$ at steady-state combustion:

$$
B_{t} \equiv \frac{u_{e}}{u_{f}} \frac{\Delta h}{h_{v}} \frac{1}{F_{R}} .
$$

In particular, if both Prandtl numbers are equal to one, $F_{R}=1$. As a result, the thermal blowing parameter becomes the same as the original one obtained by Marxman et al:

$$
B_{t}=\frac{u_{e}}{u_{f}} \frac{\Delta h}{h_{v}} .
$$

In the present study, we focus on steady combustion. In this case, the aerodynamic blowing parameter $B_{a}$ is equal to the thermal blowing parameter $B_{t}$ :

$$
B_{a}=B_{t} \text {. }
$$

The relation between the surface friction coefficient and the aerodynamic blowing parameter can be expressed approximately by the following:

$$
\frac{c_{f}}{c_{f, 0}}=q B_{a}{ }^{k},
$$

where $c_{f, 0}$ is given by the Blasius equation for turbulent boundary layers with no blowing. Marxman et al. proposed that $k$ and $q$ are 0.77 and 1.2, respectively. From the latter, Altman et al. proposed modified values of $k$ and $q$ as being 0.68 and 1.0, respectively. From Eqs. (32) and (33), we can obtain the regression rate equation, as shown below:

$$
\rho_{c} \dot{r}=0.03 q G(G x / \mu)^{-0.2} B^{1-k},
$$

where $B_{a}$ and $B_{t}$ are set to $B$. The above equation takes the same form as that obtained by Marxman et al. However, $B$ is here different from Marxman's. In order to determine the value of $B$, we must numerically solve the following equation, which is equivalent from 
Eq.(30):

$$
f\left(B, G, P r, \operatorname{Pr}_{t}, O / F, x, \mu\right) \equiv B I_{H}-\frac{\Delta h}{h_{v}}=0 .
$$

Equation (34) can be expressed in dimensionless form in terms of $G^{*}$ and $\dot{m}^{*}$. The result shows:

$$
\dot{m}^{*}=0.03 q G^{* 0.8} \operatorname{Re}_{x, \text { ref }}^{-0.2} B^{1-k} .
$$

In addition, in order to calculate regression rate characteristics, we need the ratio of the gas velocity at flame to the gas velocity in the main stream. From the diffusion-limited analysis by Marxman et al., the ratio can be written as follows:

$$
\phi_{f}=\frac{u_{f}}{u_{e}}=\frac{(O / F) B_{t}-Y_{O X, e}}{B_{t}\left\{O / F+Y_{O X, e}\right\}} .
$$

Here $O / F, B_{t}, Y_{o x, e}$ is the oxidizer-to-fuel mass ratio, the thermal blowing parameter, and the oxidizer mass fraction in the main stream, respectively.

\section{Results and Discussion}

Table 1 shows the various physical and thermodynamic properties of the PMMA/GOX hybrids. In this table, the molecular Prandtl number and the viscosity of the combustion product gas can be determined by chemical equilibrium calculations. In order to determine these values, NASA CEA-400 was used in this study ${ }^{(14)}$.

Table 1 Physical and thermodynamic properties of the PMMA/GOX hybrids

\begin{tabular}{|l|l|}
\hline Parameter & Value \\
\hline Molecular Prandtl number, $P r$ & 0.66 \\
\hline Turbulent Prandtl number, $P r_{t}$ & $1.4 \sim 1.9$ (Senda et al.) \\
\hline$\Delta h / h_{v}$ & 5.5 (Marxman et al.) \\
\hline Viscosity of combustion product gas, $\mu$ & $1.07 \times 10^{-5}[\mathrm{~Pa} \mathrm{sec}]$ \\
\hline Port diameter & $40[\mathrm{~mm}]$ \\
\hline Combustion port length & $500[\mathrm{~mm}]$ \\
\hline Mass flow rate of gaseous oxygen & $91.1[\mathrm{~g} / \mathrm{sec}]$ \\
\hline Total mass flow rate of fuel (PMMA) & $65.9[\mathrm{~g} / \mathrm{sec}]$ \\
\hline Oxidizer-to-fuel mass ratio, $O / F$ & 1.39 \\
\hline Axial distance along the port, $x$ & $90[\mathrm{~mm}]$ \\
\hline Density of PMMA & $1190\left[\mathrm{~kg} / \mathrm{m}^{3}\right]$ \\
\hline
\end{tabular}

The turbulent Prandtl number was evaluated using the experimental data of the non-reacting turbulent boundary layer with blowing. With regard to the turbulent boundary layer with blowing, many associated studies have been done. We used the experimental data compiled by Senda et al ${ }^{(15)} . \Delta h / h_{v}$ in this table represents the ratio of the enthalpy difference between the flame and the fuel surface to the summation of the latent heat and the increase of internal energy in the solid phase. For the ratio, we used the value estimated by Marxman et al ${ }^{(1)}$. For the mass flow rate of the oxidizer, the oxidizer-to-fuel mass ratio and the combustion port diameter, we referred to the experiments conducted by Yuasa et al ${ }^{(7-8)}$. The local Reynolds number is expressed by the distance parallel to the fuel surface: $x$. In the present analysis, $\mathrm{x}$ was set to be $0.09 \mathrm{~m}$. It was determined by assuming that the local 
regression rate is nearly equal to the average regression rate ${ }^{(9)}$. Regression rate characteristics of hybrid rocket motors are normally shown as a log-log plot of regression rate versus $G$. The fuel regression rate obtained by Marxman et al. is represented by a straight line on a $\log -\log$ plot, because it is proportional to $G^{0.8}$. This is not apparent in the present model that includes the effects of the Prandtl numbers variation, because Eq. (35), which determines the value of $B$, is the implicit function of $G$. We therefore calculated fuel regression rate using the present model, and then represented the regression rate characteristics on a log-log plot. As shown in Figs. 1 and 2, the obtained lines are nearly straight. It means that the calculated regression rate can be approximately expressed by a power function of $G$.

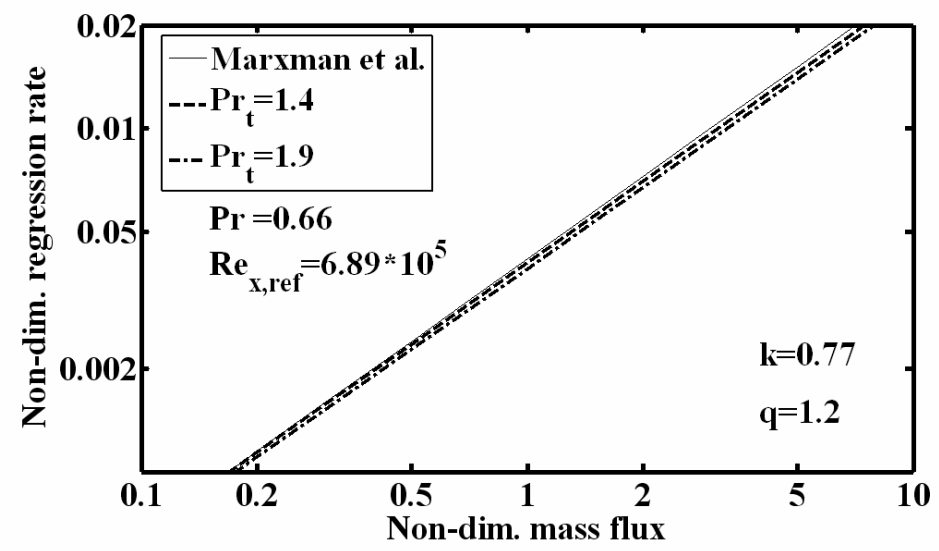

Fig. 1 A log-log plot of the fuel regression rate vs. total mass flux $(P r=0.66)$

Figure 1 shows the relation between the mass flux at a combustion port and the associated fuel regression rate on a log-log plot. This figure was obtained by calculating Eq. (36). In particular, the effects of the turbulent Prandtl number on the fuel regression rate were examined. In this figure, the molecular Prandtl number is assumed to be 0.66 . The calculated regression rate using the analysis by Marxman et al. is expressed by the narrow solid line. The other lines were obtained by using the present model. When the turbulent Prandtl number is 1.4 , the regression rates are indicated by the dashed line. When the turbulent Prandtl number is 1.9 , the regression rates are indicated by the alternating long and short dashed line. As shown in this figure, we found that fuel regression rate decreases as the turbulent Prandtl number increases.

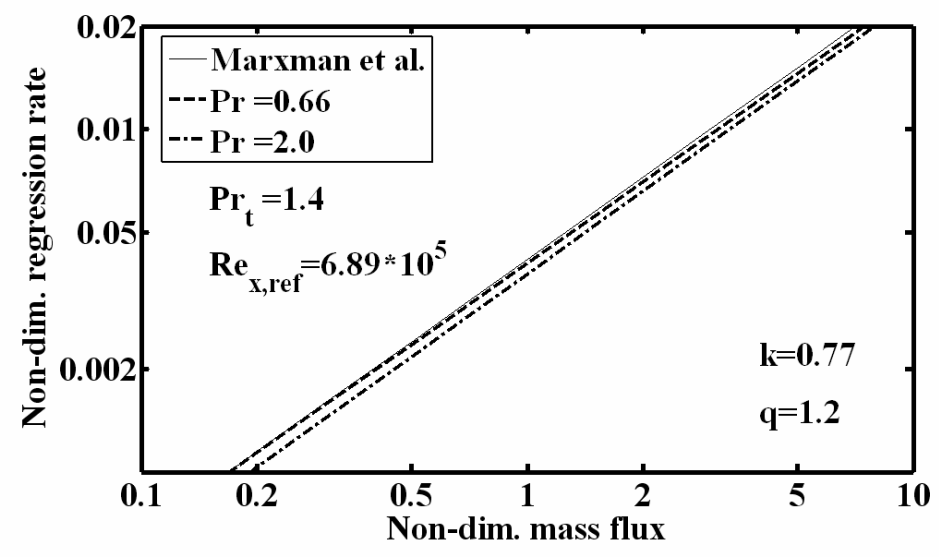

Fig. 2 A log-log plot of the fuel regression rate vs. total mass flux $\left(\operatorname{Pr}_{t}=1.4\right)$ 
Figure 2 also shows the relation between the mass flux at a combustion port and the associated fuel regression rate on a log-log plot. In particular, the effects of the molecular Prandtl number on fuel regression rate were examined. Here the turbulent Prandtl number is assumed to be 1.4. As shown in this figure, we found that fuel regression rate decreases as the molecular Prandtl number increases.

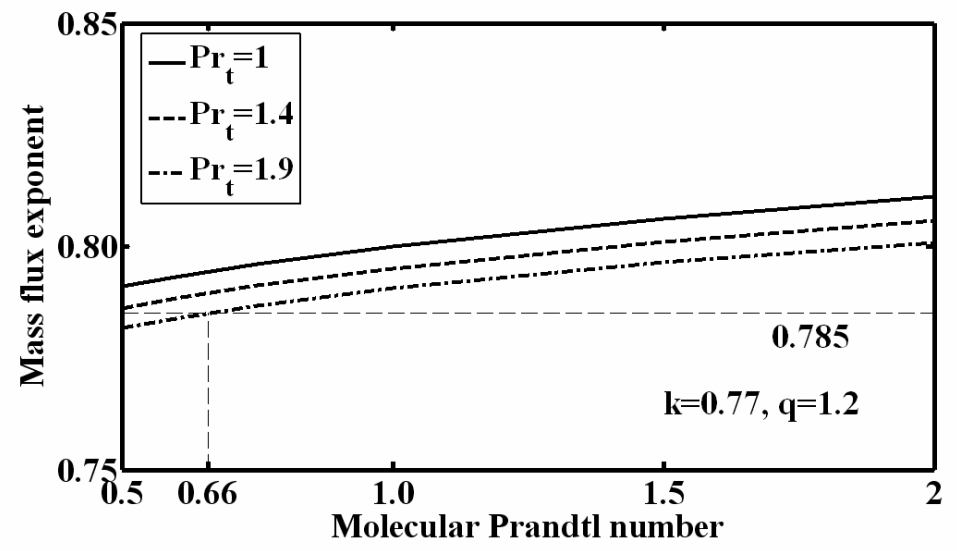

Fig. 3 Effects of the Prandtl numbers on a mass flux exponent

From many experimental results, fuel regression rate can generally be written by the following equation.

$$
\dot{r}=a G^{n},
$$

where $a$ and $n$ are the regression rate coefficient and the mass flux exponent, respectively. Calculating Eq. (36) and then comparing the calculation result with Eq. (38), we can obtain the mass flux exponent and the regression rate coefficient. Figure 3 shows the effects of the Prandtl numbers on the mass flux exponent of fuel regression rate. As shown in this figure, mass flux exponent decreases as the molecular Prandtl number decreases. In the PMMA/GOX experiments referred to in the present study, the molecular Prandtl number is 0.66. Experiments for non-reacting turbulent boundary layers with blowing have been conducted by many researchers. In this study, we used the experimental data obtained by Senda et al. The typical turbulent Prandtl number shows values in the range of 1.4 to 1.9. From the above calculations for the fuel regression rate, the expected mass flux exponent is consistent with the experimental value $(0.785)$ of the mass flux exponent obtained by Marxman et al.

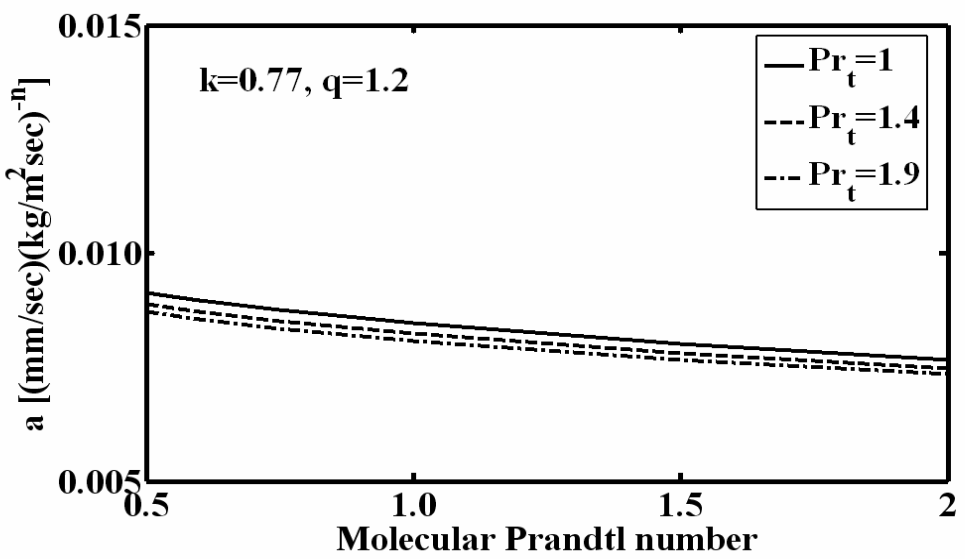

Fig. 4 Effects of the Prandtl numbers on a regression rate coefficient 
Figure 4 shows the effects of the Prandtl numbers on the regression rate coefficient of fuel regression rates. As shown in this figure, we found that the regression rate coefficient decreases as the molecular and/or turbulent Prandtl numbers increases.

As mentioned previously, the values of $\mathrm{k}$ and $\mathrm{q}$ were obtained by several researchers. However, when the Prandtl numbers are not equal to one, the effects of $\mathrm{k}$ and $\mathrm{q}$ on fuel regression rate have not been made clear. We therefore examined these effects. The results show in the following figures.

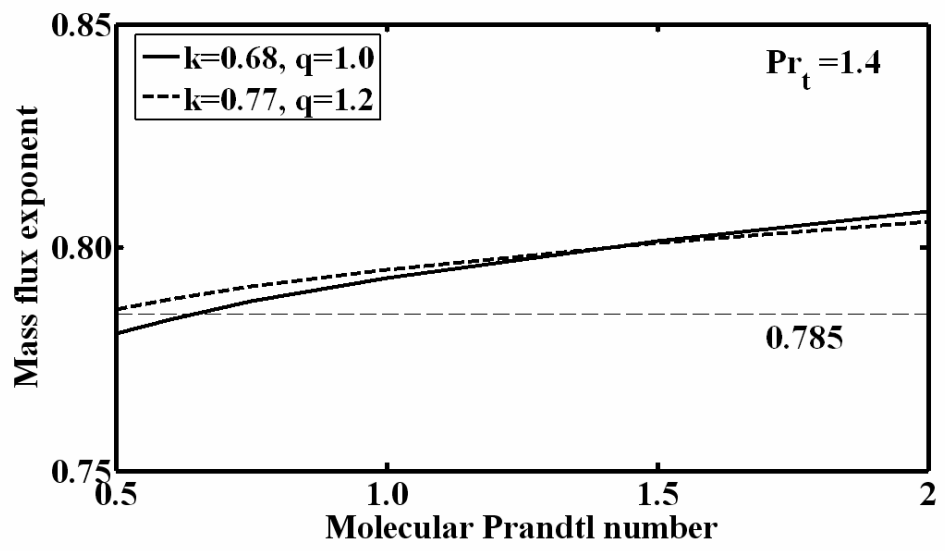

Fig. 5 Effects of $k$ and $q$ on a mass flux exponent

Figure 5 shows the effects of $k$ and $q$ on the mass flux exponent of fuel regression rates. In this figure, the solid line was obtained by using the constants proposed by Altman et al. On the other hand, the dashed line was obtained by using the constants proposed by Marxman et al. As shown in this figure, the calculated mass flux exponent using the constants proposed by Altman et al. is less than that using the constants obtained by Marxman et al. when the molecular Prandtl number is less than one.

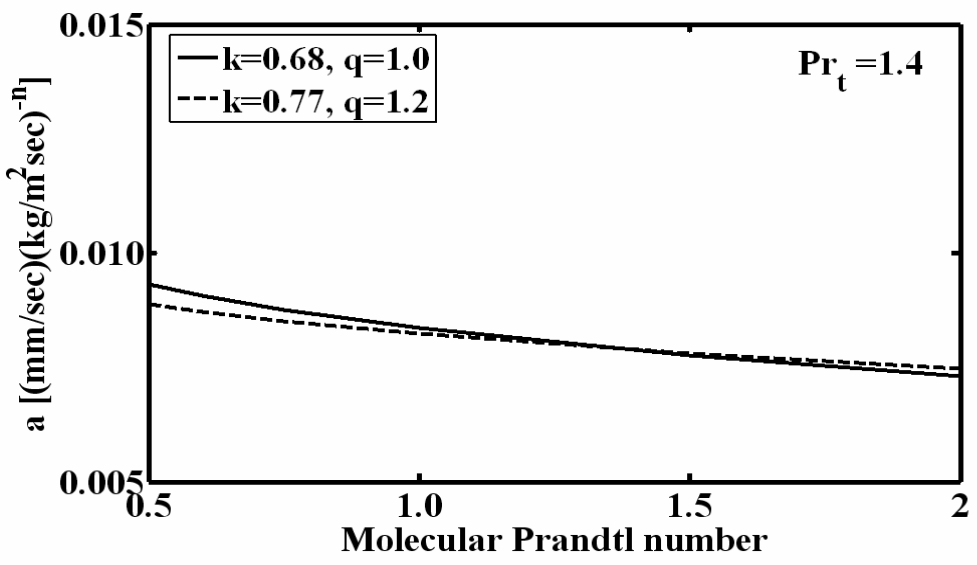

Fig. 6 Effects of $k$ and $q$ on a regression rate coefficient

Figure 6 shows the effects of $k$ and $q$ on the regression rate coefficient for hybrids. As shown in this figure, the calculated regression rate coefficient using the constants proposed by Altman et al. is greater than that using the constants obtained by Marxman et al. when the molecular Prandtl number is less than one. 


\section{Conclusion}

Fuel regression characteristics for hybrid rocket motors were obtained using the present analogy that allows for the Prandtl number variations. Consequently, the results show as follows: The mass flux exponent of fuel regression rates decreases as the molecular and/or turbulent Prandtl number decreases. The regression rate coefficient of fuel regression rates increases as the molecular and/or turbulent Prandtl number decreases. The calculated mass flux exponent using $k$ and $q$ that was proposed by Altman et al. is less than that obtained by Marxman et al. when the molecular Prandtl number is less than one. The calculated regression rate coefficient using $k$ and $q$ that was proposed by Altman et al. is greater than that obtained by Marxman et al. when the molecular Prandtl number is less than one.

\section{Acknowledgements}

This research is supported by the Hybrid Rocket Research Working Group (HRrWG) of the Institute of Space and Astronautical Science, Japan Aerospace Exploration Agency. The authors thank the members of HRrWG for helpful discussions.

\section{Appendix}

Equation (16) can be integrated using integration by parts. The result shows:

$$
\begin{aligned}
I_{H} & =P r_{t} \frac{u_{f}}{u_{e}}+\left(P r-P r_{t}\right) \int_{0}^{\delta_{f} / \delta} \frac{1}{1+c_{H} \eta^{1+\ell}} \frac{d \phi}{d \eta} d \eta \\
& =P r_{t} \frac{u_{f}}{u_{e}}+\left(P r-P r_{t}\right)\left[\frac{u_{f} / u_{e}}{1+c_{H}\left(\delta_{f} / \delta\right)^{1+\ell}}+\int_{0}^{\delta_{f} / \delta} \frac{(1+\ell) c_{H} \eta^{\ell} \phi}{\left(1+c_{H} \eta^{1+\ell}\right)^{2}} d \eta\right] \\
& =P r_{t} \frac{u_{f}}{u_{e}}+\left(P r-P r_{t}\right)\left[\frac{u_{f} / u_{e}}{1+c_{H}\left(\delta_{f} / \delta\right)^{1+\ell}}+\frac{1}{\left(\delta_{f} / \delta\right)_{c_{H}}} \int_{0}^{1} \frac{x^{\ell^{\prime}}}{(\beta+x)^{2}} d x\right],
\end{aligned}
$$

where $\beta=\left\{c_{H} \phi_{f}^{1 / \ell^{\prime}}\right\}^{-1}$.

In order to perform this integration, we define the following integral:

$$
M\left(\beta, \ell^{\prime}\right) \equiv \int_{0}^{1} \frac{x^{\ell^{\prime}}}{(\beta+x)^{2}} d x .
$$

This integral can be integrated approximately, as shown below:

$$
M\left(\beta, \ell^{\prime}\right) \approx \int_{0}^{1} \frac{x^{\ell^{\prime}}}{(\beta+x)^{2+\ell^{\prime}}} d x=\frac{1}{\left(1+\ell^{\prime}\right) \beta}\left(\frac{1}{1+\beta}\right)^{1+\ell^{\prime}} .
$$

However, the calculation accuracy of the integral value performed using this approximation becomes worse in the range of the value $\beta$ used in this study. Therefore, we performed the integration with the appropriate value $\beta_{0}$ instead of $\beta$. Then the integral value was transformed into a value that would be obtained using the original $\beta$. The detailed calculations are shown below. Rearranging Eq. (A3) by integration by parts,

$$
\begin{aligned}
M & =\int_{0}^{1} \frac{x^{\ell^{\prime}}}{(\beta+x)^{2}} d x=\frac{1}{\left(1+\ell^{\prime}\right)(1+\beta)^{2}}+\frac{2}{1+\ell^{\prime}} \int_{0}^{1} \frac{x^{1+\ell^{\prime}}}{(\beta+x)^{3}} d x \\
& =\frac{1}{\left(1+\ell^{\prime}\right)(1+\beta)^{2}}+\frac{2}{1+\ell^{\prime}} \int_{0}^{1} \frac{x^{\ell^{\prime}}}{(\beta+x)^{2}} d x-\frac{2 \beta}{1+\ell^{\prime}} \int_{0}^{1} \frac{x^{\ell^{\prime}}}{(\beta+x)^{3}} d x .
\end{aligned}
$$


Differentiating Eq. (A3) with respect to $\beta$,

$$
\frac{d M}{d \beta}=-2 \int_{0}^{1} \frac{x^{\ell^{\prime}}}{(\beta+x)^{3}} d x
$$

Substituting Eq. (A6) into Eq. (A5) and rearranging it, we can obtain the following differential equation:

$$
\frac{d M}{d \beta}+\frac{1-\ell^{\prime}}{\beta} M=-\frac{1}{\beta(1+\beta)^{2}} .
$$

Solving the above differential equation, the result shows,

$$
M\left(\beta, \ell^{\prime}\right)=-\frac{1}{\beta^{1-\ell^{\prime}}} \int \frac{1}{\beta^{\ell^{\prime}}(1+\beta)^{2}} d \beta .
$$

This integral can be integrated approximately, as shown below:

$$
M\left(\beta, \ell^{\prime}\right) \approx-\frac{1}{\beta^{1-\ell^{\prime}}} \int \frac{1}{\beta^{\ell^{\prime}}(1+\beta)^{2-\ell^{\prime}}} d \beta=-\frac{1}{1-\ell^{\prime}}\left(\frac{1}{1+\beta}\right)^{1-\ell^{\prime}}+\frac{C}{\beta^{1-\ell^{\prime}}},
$$

where $C$ is an integration constant. Substituting $\beta_{0}$ into $\beta$, and rearranging for $C$, we can obtain the following equation:

$$
C=\frac{1}{1-\ell^{\prime}}\left(\frac{\beta_{0}}{1+\beta_{0}}\right)^{1-\ell^{\prime}}+\beta_{0}^{1-\ell^{\prime}} M\left(\beta_{0}, \ell^{\prime}\right) .
$$

Here, $M\left(\beta_{0}, \ell^{\prime}\right)$ in Eq. (A10) is approximately estimated by using Eq. (A4). Actually, $\beta_{0}$ is allowed to be an arbitrary value. However, $\beta_{0}$ must take an appropriate value, because we use the approximate calculation in order to obtain Eq. (A4) and Eq. (A9). In this study, $\beta_{0}$ is assumed to be 2.0. Substituting Eq. (A10) into Eq. (A9) yields

$$
M\left(\beta, \ell^{\prime}\right)=-\frac{1}{1-\ell^{\prime}}\left(\frac{1}{1+\beta}\right)^{1-\ell^{\prime}}+\frac{1}{\beta^{1-\ell^{\prime}}}\left\{\frac{1}{1-\ell^{\prime}}\left(\frac{\beta_{0}}{1+\beta_{0}}\right)^{1-\ell^{\prime}}+\beta_{0}^{1-\ell^{\prime}} M\left(\beta_{0}, \ell^{\prime}\right)\right\} .
$$

When Eq. (A2) is substituted into the above equation, Eq. (A11) is rewritten in Eq. (A12):

$$
M\left(\left\{c_{H} \phi_{f}{ }^{1 / \ell^{\prime}}\right\}^{-1}, \ell^{\prime}\right)=\frac{c_{H}{ }^{1-\ell^{\prime}} \phi_{f}{ }^{1 / \ell^{\prime}-1}}{1-\ell^{\prime}}\left\{-\frac{1}{C_{1}^{1-\ell^{\prime}}}+\left(\frac{\beta_{0}}{1+\beta_{0}}\right)^{1-\ell^{\prime}}+\left(1-\ell^{\prime}\right) \beta_{0}{ }^{1-\ell^{\prime}} M\left(\beta_{0}, \ell^{\prime}\right)\right\} .
$$

Substituting the above equation into Eq. (A1), we can obtain the following equation:

$$
I_{H}=\phi_{f} F_{R}
$$

where $F_{R}=P r_{t}+\left(P r-P r_{t}\right)\left\{\frac{1}{C_{1}}+\frac{C_{2}}{\left(1-\ell^{\prime}\right) c_{H}^{\ell^{\prime}} \phi_{f}}\right\}$.

\section{References}

(1) Marxman, G.A., Wooldridge, C.E., and Muzzy, R.J., Fundamentals of Hybrid Boundary Layer Combustion, Progress in Astronautics and Aeronautics, Vol.15 (1964), pp. 485-521. (2) Marxman, G.A., Combustion in the Turbulent Boundary Layer on a Vaporizing Surface, Tenth Symposium (International) on Combustion (1965), pp. 1337-1349.

(3) Marxman, G.A., and Gilbert, M., Turbulent Boundary Layer Combustion in the Hybrid Rocket, Ninth International Symposium on Combustion (1963), pp. 371-383.

(4) Karabeyoglu, M.A., De Zilwa, S., Cantwell, B. J., and Zilliac, G., Transient Modeling of Hybrid Rocket Low Frequency Instabilities, Journal of Propulsion and Power, Vol. 21, No. 6,

Nov.-Dec. (2005), pp. 1107-1117. 
(5) Boardman, T. A., Alternative Interpretations of Boundary Layer Blowing Coefficient in Chapter 15, Rocket Propulsion Elements, 7th ed., John Wiley \& Sons (2001), pp. 737-738.

(6) Yuasa, S., Ide, T., Masugi, M., Sakurai, T., Shiraishi, N., and Shimada, T., Visualization and Emission Spectra of Flames in Combustion Chamber of Swirling-Oxidizer-Flow-Type Hybrid Rocket Engines, Journal of Thermal Science and Technology, Vol.6, No.2 (2011), pp. 268-277. (7) Tamura, T., Yuasa, S., and Yamamoto, K., Effects of Swirling Oxidizer Flow on Fuel Regression Rate of Hybrid Rockets, AIAA Paper 99-2323 (1999).

(8) Yuasa, S., Shimada, O., Imamura, T., Tamura, T., and Yamamoto, K., A Technique for Improving the Performance of Hybrid Rocket Engines, AIAA Paper 99-2322 (1999). (9) Yuasa, S., Yamamoto, K., Hachiya, H., Kitagawa, K., and Oowada, Y., Development of a Small Sounding Hybrid Rocket with a Swirling-Oxidizer-Type Engine, AIAA Paper 20013537 (2001).

(10) Morita, T., A Combustion Response Function of Hybrid Propellants on the basis of the Analogy between Heat and Momentum Transfer (in Japanese), Proceedings of 54th Space Sciences and Technology Conference, JSASS-2009-4227 (2009).

(11) Morita, T., A Study on Oscillatory Combustion in Hybrid Rockets (in Japanese), Proceedings of Space Transportation Symposium, STCP-2009-72 (2010).

(12) Lengelle, G., Model Describing the Erosive Combustion and Velocity Response of Composite Propellants, AIAA Journal, Vol.13, No.3 (1975), pp. 315-322.

(13) Karabeyoglu, A., Transient Combustion in Hybrid Rockets, Ph.D. Dissertation, Stanford Univ., Dept. of Aeronautics and Astronautics, Aug. (1998).

(14) McBride, B.J., and Gordon, S., Computer Program for Calculation of Complex Chemical Equilibrium Compositions and Applications, NASA RP 1311 (1994).

(15) Senda, M., Suzuki, K., and Sato, T., Turbulence Structure Related to the Heat Transfer in a Turbulent Boundary Layer with Injection, Proceedings of 2nd International Symposium on Turbulent Shear Flows (1979), pp. 917-922. 